\title{
Problemas de coordenação motora e de atenção em crianças em idade escolar
}

\section{Motor coordination and attention problems in school-aged children}

\author{
Annyelle Santos Franca ${ }^{1}$, Ana Amélia Cardoso ${ }^{2}$, Clarice Ribeiro Soares Araújo ${ }^{3}$
}

http://dx.doi.org/10.11606/issn.2238-6149.v28i1p86-92

Franca AS, Cardoso AA, Araújo CRS. Problemas de coordenação motora e de atenção em crianças em idade escolar. Rev Ter Ocup Univ São Paulo. 2017 jan.-abr.;28(1):86-92.

RESUMO: Objetivos: Investigar a prevalência de dificuldades de coordenação motora em crianças de 7 e 8 anos, em escolas municipais de João Pessoa/PB; e verificar se há associação entre dificuldades motoras e déficit de atenção e hiperatividade e nível socioeconômico. Método: Foram avaliadas 535 crianças com o Developmental Coordination Disorder Questionnaire (DCDQ-Brasil), o Questionário Classificação Econômica Brasil e o Swanson, Nolan and Pelham IV Scale (SNAP-IV). Estatística descritiva foi utilizada para caracterizar amostra e teste qui-quadrado foi utilizado para verificar associação entre as variáveis. Resultados: Respostas dos pais ao DCDQ-Brasil apontam que 253 crianças (47,2\%) apresentam dificuldades de coordenação motora. Foi observada associação das dificuldades de coordenação motora com déficit de atenção e hiperatividade, e com o nível socioeconômico. Conclusões: Resultados apontam prevalência considerável de problemas de coordenação motora em crianças de 7 e 8 anos, e associação desses problemas com TDAH e com nível socioeconômico.

DESCRITORES: Transtornos das habilidades motoras; Transtorno do déficit de atenção com hiperatividade; Prevalência; Classe social; Criança.
Franca AS, Cardoso AA, Araújo CRS. Motor coordination and attention problems in school-aged children. Rev Ter Ocup Univ São Paulo. 2017 Jan.-Apr.;28(1):86-92.

ABSTRACT: Objectives: To investigate the prevalence of motor coordination difficulties in school-aged children with 7 and 8 years old in municipal schools of João Pessoa/PB; to check whether there is an association between the motor difficulties with attention deficit hyperactivity disorder and socioeconomic status. Method: 535 children were evaluated with the Developmental Coordination Disorder Questionnaire (DCDQ-Brazil), the Questionnaire Economic Classification Brazil and the Swanson, Nolan and Pelham Scale IV - SNAP-IV. Descriptive statistics characterized the sample and chi-square test assessed the association between variables. Results: According to parents' responses to DCDQ-Brazil, 253 children (47.2\%) have difficulties in motor coordination. It was observed association of the motor difficulties with attention deficit hyperactivity disorder, and socioeconomic status. Conclusions: The results show high prevalence of motor coordination problems in children aged 7 and 8 , and association of the difficulties of coordination with attention deficits and hyperactivity and socioeconomic status.

KEYWORDS: Motor skills disorders; Attention deficit hyperactivity disorder with hyperactivity; Prevalence; Social class; Child.

Este trabalho é proveniente do Trabalho de Conclusão do Curso de Terapia Ocupacional da primeira autora. Foi apresentado na XI International Conference on Developmental Coordination Disorder em Toulouse, França, 2015.

1. Terapeuta Ocupacional, Governo da Paraíba, Hospital Regional de Emergência e Trauma Dom Luiz Gonzaga Fernandes. E-mail: annyellesfranca@gmail.com.

2. Terapeuta Ocupacional, Professora Adjunta, Universidade Federal de Minas Gerais, Departamento de Terapia Ocupacional da Escola de Educação Física, Fisioterapia e Terapia Ocupacional, Campus Pampulha. E-mail: anaameliacardoso@gmail.com.

3. Terapeuta Ocupacional, Professora Adjunta, Universidade Federal da Paraíba, Departamento de Terapia Ocupacional, Centro de Ciências da Saúde - Campus I.E-mail: clariceribeiro@hotmail.com.

Endereço para correspondência: Clarice Ribeiro Soares Araújo. Rua José de Melo, 90, apart. 404 - Santa Rosa, Belo Horizonte, MG. CEP: $31255-490$. 


\section{INTRODUÇÃO}

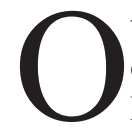

transtorno do desenvolvimento da coordenação (TDC), de acordo com o Manual Diagnóstico e Estatístico de Transtornos Mentais ${ }^{1}$, apresenta-se como disfunção significativa nas habilidades motoras, que pode ser observada na criança através da dificuldade de coordenar movimentos, sem sinais aparentes de lesão cerebral e causas neurológicas ou sensoriais. Geralmente a criança tem inteligência normal, mas os problemas de coordenação motora grossa e fina podem interferir no rendimento escolar e em atividades de vida diária (AVD) $)^{1,2}$.

Os traços mais comuns de se observar nessas crianças são problemas na habilidade motora fina, função motora grossa, coordenação geral e controle durante a execução do movimento, dificuldade para executar atividades típicas da infância como jogar bola, escrever, andar de bicicleta, vestir roupas, amarrar cadarços e utilizar tesoura, talheres e outros utensílios domésticos, atrapalhando desta forma a execução das AVD, atividades de brincar e tarefas escolares ${ }^{2,3,4,5}$. Além dos problemas de coordenação motora que refletem no cotidiano da criança, podem ser percebidas co-ocorrências associadas como a obesidade, problemas de saúde mental e o transtorno do déficit de atenção e hiperatividade (TDAH), podendo trazer mais prejuízos funcionais ${ }^{3,6,7,8,9}$.

Estudos epidemiológicos indicam que a prevalência de TDC pode variar de 5 a $9 \%$ em crianças em idade escolar, sendo que a maior parte delas é do sexo masculino, não importando o nível socioeconômico ${ }^{10}$. A prevalência do TDC e sua relação com outras co-ocorrências e estilos de vida vem sendo estudada por diversos autores em todo o mundo.

No Brasil, alguns estudos vêm investigando a prevalência do TDC em crianças com idade escolar. Silva e Beltrame ${ }^{11}$ identificaram a prevalência de $11 \%$ problemas motores em 406 crianças entre 7 e 10 anos de uma escola municipal de São José/SC, região sul do país. Já França ${ }^{12}$ encontrou a prevalência de $10,8 \%$ de problemas motores em estudo na cidade de Florianópolis/SC, com 417 crianças avaliadas com o Movement Assesment Battery for Children (MABC). Ainda em 2012, foi realizado por Valentini et al. ${ }^{13}$ outro estudo na região Sul do Brasil (Rio Grande do Sul, Paraná e Santa Catarina) com 1.587 crianças entre 04 e 12 anos de idade, que foram avaliadas com o MABC, ao final chegou ao resultado que $19,9 \%$ das crianças são prováveis TDC e outros $16,8 \%$ tem risco de serem acometidos pelo transtorno. Na região centro- oeste do país, Melo ${ }^{14}$ realizou um estudo no município de Ceilândia-DF, e observou que $8 \%$ da população estudada tinha TDC.

No município de Araraquara/SP, Jóia ${ }^{15}$ avaliou 101 crianças com 07 anos com o objetivo de identificar a prevalência do TDC, possível relação deste com a classificação socioeconômica da família e sinais de TDAH. Para isso, utilizou o MABC, Swanson, Nolan and Pelham IV Scale (SNAP IV) e o Critério Padrão de Classificação Econômica Brasil (CPCEB) e identificou a prevalência de $2,97 \%$ de TDC severo e $4,95 \%$ de crianças com TDC moderado, além de terem sido encontrados dados que mostraram relação direta entre o TDC moderado e o TDAH.

No estudo conduzido por Cardoso et al. ${ }^{16}$ um total de 793 crianças foi triado pelo Developmental Coordination Disorder Questionnaire (DCDQ-Brasil); 90 foram identificadas como provável TDC; 91 controles pareados foram selecionados entre os participantes restantes. As crianças de ambos os grupos foram avaliadas com o MABC-2 e o SNAP-IV e os pesquisadores observaram que 34 crianças foram classificadas como prováveis TDC, conforme definido por uma combinação da DCDQ-Brasil e MABC-2 pontos (percentil 5). A frequência final do TDC, entre crianças de 7 e 8 anos foi de $4,3 \%$.

Percebe-se que no Brasil, o teste mais utilizado para identificar crianças com TDC tem sido o MABC-2 - especialmente em pesquisas -, cujo primeiro estudo de validação é recente ${ }^{17}$. Entretanto, para a realidade brasileira, o acesso a testes estrangeiros é difícil, a importação tem alto custo, e, também, ainda não temos normas brasileiras para utilizá-los com segurança. Desta forma, no nosso país, é comum o uso de questionários de triagem para pais e professores, tanto pela boa relação custo benefício, quanto pelo fato deste público estar presente grande parte do tempo na vida da criança e serem bons informantes ${ }^{3,8,9}$.

Na revisão bibliográfica para o presente estudo, não foram encontrados artigos que abordassem se o TDC tem alguma relação com o nível socioeconômico da família, apesar dos dados da Associação Americana de Psiquiatria indicarem que o TDC não tem relação com o nível socioeconômico.

No nordeste do Brasil, ainda não existem estudos que investigaram a prevalência de problemas motores - como o TDC - em crianças na idade escolar. Assim, os objetivos do presente estudo foram (a) identificar a prevalência de crianças com sinais de problemas motores de 7 e 8 anos, matriculadas nas escolas públicas do município de João Pessoa/PB e (b) verificar se há associação das dificuldades de coordenação motora com TDAH, com nível socioeconômico e com sexo das crianças. 


\section{MÉTODO}

Estudo descritivo de corte transversal, cujos participantes foram crianças entre 7 e 8 anos sem diagnóstico prévio de alguma síndrome ou deficiência, que frequentavam o ensino fundamental de escolas da rede pública de ensino do município de João Pessoa, Paraíba, Brasil, no ano de 2014.

A cidade de João Pessoa tem população de 780.738 habitantes, é considerada uma cidade de porte médio, o índice de desenvolvimento humano municipal (IDHM) é de 0,763 (alto desenvolvimento humano) e tem $14 \%$ de taxa de analfabetismo. É a capital do estado da Paraíba, que tem 3.943.885 habitantes; IDHM de 0,658, um dos cinco piores índices das 27 unidades federativas. As secretarias municipal e estadual de educação deram consentimento para que a pesquisa fosse realizada nas escolas com ensino fundamental. A pesquisa foi aprovada pelo Comitê de Ética em Pesquisa do Centro de Ciências da Saúde da Universidade Federal da Paraíba sob parecer $\mathrm{N}^{\circ}$ 0231/14, CAAE: 30997014.2.0000.5188. O total de 95 escolas foram convidadas a colaborar na pesquisa, por meio dos diretores. Posteriormente, kits de avaliação foram entregues às professoras e pais ou responsáveis pelas crianças que tiveram um prazo de 15 dias para devolverem o material, caso aceitassem participar da pesquisa de forma voluntária, assinando o Termo de Consentimento Livre e Esclarecido e o Termo de Assentimento.

\section{Instrumentos}

O Questionário da História do Desenvolvimento, elaborado pelas pesquisadoras, teve por finalidade investigar a história da criança a partir da concepção, gravidez e nascimento.

O Developmental Coordination Disorder Questionnaire (DCDQ) ${ }^{18}$ é um questionário para pais utilizado na identificação de sintomas de problemas de coordenação motora leve, de fácil aplicação, específico para triagem que recentemente foi traduzido e adaptado para o português, resultando no DCDQBrasil $^{19}$, versão usada no presente estudo. Após alguns ajustes, a versão brasileira atingiu bons índices psicométricos (sensibilidade 0,73 , confiabilidade testereteste 0.97 , consistência interna 0,92 ), indicando ser útil para distinguir crianças com e sem transtorno do desenvolvimento da coordenação.

O DCDQ-Brasil compreende 15 itens distribuídos nas seguintes sessões: controle durante o movimento, habilidades motoras finas e de escrita, habilidades motoras grossas e de planejamento, coordenação motora global. Os pais devem levar em consideração o desempenho da criança em relação a outras da mesma idade; os itens são pontuados em escala de cinco pontos $(1=$ "não é nada parecido com sua criança" e 5 = "extremamente parecido com sua criança"), com pontuação máxima igual a 75 ; quanto maior a pontuação, melhor o desempenho motor da criança.

Embora ainda não haja normas brasileiras para o DCDQ-Brasil, foram utilizados os pontos de corte propostos para crianças canadenses, pois as médias de desempenho são similares nos dois países ${ }^{19}$. Suspeitase que a criança tenha TDC se: de cinco anos a sete anos e onze meses obtiver escore total de $0-46$; de oito anos aos nove anos e onze meses, obtiver escore total de $0-55$; de 10 anos a 15 anos e seis meses, obtiver escore total de $0-57$

O Questionário Critério de Classificação Econômica Brasil (CCEB) da Associação Brasileira de Empresas de Pesquisa (ABEP) ${ }^{20}$ foi utilizado para fins de classificação do nível econômico das famílias, respondido pelos cuidadores. O CCEB possui dados com base no levantamento sócio econômico de 2011 do Instituto Brasileiro de Opinião e Estatística (IBOPE) com fins de estimar o poder de compra das famílias, abandonando a pretensão de classificar a população em termos de "classes sociais" ${ }^{20}$. O questionário abrange perguntas da quantidade de eletrodomésticos, automóveis e cômodos na casa, além do nível de escolaridade do chefe da família, sendo possível classificar o nível econômico em A1, A2, B1, B2, $\mathrm{C} 1, \mathrm{C} 2$, D e E.

Já as professoras, responderam ao Swanson, Nolan and Pelham IV Scale - SNAP-IV ${ }^{21}$, que é um questionário de domínio público usado para triagem do Transtorno do Déficit de Atenção e Hiperatividade (TDAH). O SNAP-IV

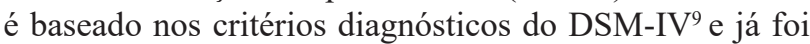
traduzido para o português ${ }^{22}$.

O questionário é composto por 18 afirmativas, das quais nove se referem a comportamentos de desatenção e as demais a comportamentos relacionados à hiperatividade; as opções de resposta são "Nem um pouco", "Só um pouco", "Bastante" e "Demais". Se a criança obtiver pelo menos seis alternativas marcadas como "Bastante" ou "Demais" nas nove primeiras afirmativas, ela tem mais sinais de desatenção que o esperado; enquanto que se ela obtiver seis pontos marcados como "Bastante" ou "Demais" nas nove últimas, considera-se que tenha mais características de hiperatividade/impulsividade que o esperado. 
Franca AS, et al. Problemas de coordenação motora e de atenção em crianças. Rev Ter Ocup Univ São Paulo. 2017 jan./abr.;28(1):86-92.

\section{Coleta de Dados}

Para dar início à coleta de dados foi utilizada a lista das escolas municipais disponível no site da prefeitura, que constava de 95 escolas. As escolas foram agrupadas por bairros para facilitar o processo de entrega dos kits. A coleta teve início dia 13 de agosto de 2014, iniciando pelos bairros mais distantes e de difícil acesso.

As visitas foram realizadas pela equipe do grupo de pesquisa composto pela pesquisadora e por três voluntárias estudantes de graduação de terapia ocupacional, que foram treinadas anteriormente para essa atividade. Durante as abordagens aos diretores, grande parte se mostrou interessada em permitir que a escola e seus alunos participassem da pesquisa, e, logo em seguida, conduziam as pesquisadoras para conversar com as professoras do $2^{\circ}$ ano para que seu papel no estudo fosse explicado.

Os questionários foram entregues aos pais ou responsáveis legais pelas crianças para o auto preenchimento. A devolução ocorreu 30 dias após a entrega, para que fosse realizada a pontuação dos testes que contou com a ajuda de outras duas voluntárias do projeto de pesquisa para posterior análise dos dados.

\section{Análise dos dados}

O Statistical Package for Social Sciences (SPSS) versão 18.0 foi utilizado para análise descritiva dos dados coletados. Teste qui-quadrado $\left(\mathrm{X}^{2}\right)$ foi utilizado para verificar associação entre o resultado no DCDQBrasil e o nível socioeconômico, resultado no SNAP-IV e sexo. Para todas as análises foi considerado nível de significância de 5\%.

\section{RESULTADOS}

Das 95 escolas abordadas, 23 não participaram da pesquisa, por se encaixar em um dos seguintes critérios: (a) difícil acesso da equipe à escola; (b) a escola não existe mais; (c) se tornou creche; (d) não tem alunos do $2^{\circ}$ ano do Ensino Fundamental I; (e) diretor (a) ou professoras não aceitaram participar do estudo. Nas 72 escolas que participaram do estudo, foram entregues 2.818 kits que continham as avaliações para os pais e apenas $535(18,98 \%)$ os devolveram corretamente preenchidos. Dos 535 kits que retornaram preenchidos, todos apresentavam o DCDQ-Brasil, 431 (80,56\%) continham o SNAP-IV e 488 (91,21\%) continham o CCEB.

As características da amostra são apresentadas na Tabela 1.

As famílias dessas 253 crianças que obtiveram pontuação indicativa de problemas motores/possível TDC, 200 crianças $(79 \%)$ se encontram nas classes econômicas C, D, e E, podendo ser consideradas como famílias com baixo poder aquisitivo.

Nas Tabelas 2 e 3 são apresentados os resultados da análise $\mathrm{X}^{2}$, de associação entre os resultados do DCDQ-Brasil com o SNAP-IV e com o nível sócio econômico, respectivamente.

Tabela 1 - Características da amostra

\begin{tabular}{llllll}
\hline & \multirow{2}{*}{$\mathbf{N}$} & \multicolumn{2}{c}{$\mathbf{7}$ anos } & \multicolumn{2}{c}{$\mathbf{8}$ anos } \\
\cline { 3 - 6 } & 535 & $171(32 \%)$ & $159(29,7 \%)$ & $99(18,5 \%)$ & $106(19,8 \%)$ \\
\hline Total & $253(47,2 \%)$ & $64(12 \%)$ & $65(12,2 \%)$ & $53(9,9 \%)$ & $71(13,3 \%)$ \\
\hline Provável TDC & $282(52,8 \%)$ & $107(20 \%)$ & $94(17,6 \%)$ & $46(8,6 \%)$ & $35(6,5 \%)$ \\
\hline Desenvolvimento motor típico & $40(9,3 \%)$ & $16(3,7 \%)$ & $13(3 \%)$ & $6(1,4 \%)$ & $5(1,2 \%)$ \\
\hline TDAH com predomínio de desatenção* & $16(3,7 \%)$ & $2(0,5 \%)$ & $10(2,3 \%)$ & $1(0,2 \%)$ & $3(0,7 \%)$ \\
\hline TDAH com predomínio de hiperatividade* & $34(7,9 \%)$ & $4(0,9 \%)$ & $13(3 \%)$ & $4(0,9 \%)$ & $13(3 \%)$ \\
\hline TDAH combinado* & $341(79,1 \%)$ & $116(26,9 \%)$ & $94(21,8 \%)$ & $72(16,7 \%)$ & $59(13,7 \%)$ \\
\hline Sem sinais de TDAH*
\end{tabular}

Nota: *apenas 431 crianças retornaram o SNAP-IV totalmente preenchido; os dados de porcentagem se referem a esse número de crianças.

Tabela 2 - Associação entre os resultados do DCDQ-Brasil e do SNAP-IV

\begin{tabular}{lcccc}
\hline \multirow{2}{*}{ DCDQ-Brasil } & \multicolumn{4}{c}{ SNAP-IV } \\
\cline { 2 - 5 } & Sem TDAH & TDAH hiperatividade & TDAH desatenção & TDAH combinado \\
\hline Desenvolvimento típico & 194 & 9 & 10 & 12 \\
\hline Provável TDC & 147 & 7 & 30 & 22 \\
\hline Total & 341 & 16 & 40 & 34 \\
\hline
\end{tabular}

Nota: Apenas 431 crianças retornaram o SNAP-IV totalmente preenchido. 
Franca AS, et al. Problemas de coordenação motora e de atenção em crianças. Rev Ter Ocup Univ São Paulo. 2017 jan./abr.;28(1):86-92.

Tabela 3 - Associação entre os resultados do DCDQ-Brasil e Classificação Socioeconômica

\begin{tabular}{lccccccc}
\hline \multirow{2}{*}{ DCDQ-Brasil } & \multicolumn{7}{c}{ Classificação socioeconômica } \\
\cline { 2 - 7 } & A2 & B1 & B2 & C1 & C2 & D & E \\
\hline Desenvolvimento Típico & 2 & 4 & 50 & 71 & 69 & 66 & 2 \\
\hline Provável TDC & 0 & 3 & 21 & 47 & 76 & 74 & 3 \\
\hline Total & 2 & 7 & 71 & 118 & 145 & 140 & 5 \\
\hline
\end{tabular}

Nota: Apenas 488 crianças retornaram o CCEB totalmente preenchido.

\section{DISCUSSÃO}

Apesar da grande adesão das escolas ao estudo, percebe-se que o número de kits devolvidos foi inferior ao esperado, chegando a $18,98 \%$ do total de avaliações enviadas. Pode-se atribuir a isto, o fato dos pais não estarem familiarizados com procedimentos de pesquisa auto-aplicada, em que eles devem responder sem o auxílio/orientação direta do pesquisador. Outro ponto a ser levantando, é a possibilidade de dificuldade de compreensão do teste DCDQ-Brasil, que exige que os cuidadores comparem sua criança com outra da mesma idade e lhe atribuam uma pontuação para seu desempenho em determinadas tarefas. Notou-se com certa frequência que testes foram descartados pelo fato de terem tido respostas do tipo "Sim" ou "Não", o que sugere que os pais não compreenderam as orientações do cabeçalho do instrumento. Em outras afirmativas, os pais colocaram observações relatando que a criança não tivera oportunidade de desempenhar aquela tarefa. Ainda foi possível identificar que muitos pais ou responsáveis legais preencheram apenas parte dos questionários, talvez pelo baixo nível de escolaridade - muitos eram analfabetos, de acordo com dados do CCEB.

Situação semelhante foi relatada por $\mathrm{Cardoso}^{23}$, que também encontrou dificuldades para receber os questionários preenchidos pelos pais. Em sua tese de doutorado, distribuiu questionários para 1.879 crianças, mas apenas 793 (42,2\%) retornaram totalmente preenchidos e acompanhados do termo de consentimento autorizando sua participação. A autora aponta que responder questionários não faz parte da rotina da maioria dos brasileiros, e talvez muitos pais não tenham colaborado com a pesquisa porque acharam difícil responder os questionários, o que também pode explicar as dificuldades enfrentadas no presente estudo.

Prado $^{18}$ realizou a tradução e adaptação transcultural do DCDQ para a população brasileira, além de verificar algumas propriedades psicométricas do instrumento. Nesse processo, a autora identificou que algumas questões não condiziam com a cultura brasileira e criou uma nova versão do questionário com alterações, $\operatorname{Prado}^{18}$ recomenda que o teste seja utilizado com amostragem mais ampla, a fim de verificar o padrão de escores nas diferentes idades e determinar pontos de corte para triagem do TDC no Brasil. Neste aspecto, o presente estudo é pioneiro, uma vez que nenhum estudo com o DCDQ-Brasil foi realizado na região nordeste do Brasil. Além disso, considerando o tamanho da amostra, os dados podem ser úteis para colaborar com a definição dos pontos de corte para crianças brasileiras, especialmente aquelas de baixo nível socioeconômico.

A prevalência de $47,2 \%$ de possível TDC (Tabela 1), observada no presente estudo, é bem mais alta do que a relatada na literatura ${ }^{11-15}$. É importante considerar que não foi utilizado nenhum teste de observação direta do desempenho motor da criança, apenas questionários respondidos pelos pais. Cardoso e Magalhães ${ }^{24}$ recomendam que a identificação dessas crianças seja feita por meio do uso de avaliações de múltiplas fontes - triangulando informações de pais, professores e da própria criança. Futuros estudos devem incluir instrumentos de avaliação direta do desempenho motor, seguindo a recomendação dessas autoras.

Os resultados deste estudo apontam associação de provável TDC com sinais de TDAH (Tabela 2), especialmente dos tipos desatento e combinado desatenção com hiperatividade, o que também é apontado na literatura internacional ${ }^{3,6-9}$. Em estudo realizado no Brasil, Cardoso et al. ${ }^{16}$ observaram que, considerando a frequência de TDAH, com base em respostas de professores ao SNAP-IV, dentro do grupo TDC, 41,2\% mostraram sinais de TDAH: seis crianças com TDC $(17,6 \%)$ exibiam sinais de déficit de atenção, uma $(2,9 \%)$ apresentou sinais de hiperatividade/impulsividade, sete $(20,7 \%)$ apresentaram sinais de TDAH combinado, 18 $(52,9 \%)$ não apresentaram sinais de TDAH e dois $(5,9 \%)$ não retornaram os questionários. Essa associação entre dificuldades motoras e TDAH aponta a necessidade de se fazer o diagnóstico o mais precocemente possível e oferecer tratamento adequado às crianças, uma vez que as comorbidades aumentam as chances de fracasso escolar e problemas emocionais secundários ${ }^{7,9}$. 
A associação significativa de provável TDC com baixo nível socioeconômico (Tabela 3) sugere que o ambiente pode ter influência no desenvolvimento motor, uma vez que crianças de famílias com baixo poder aquisitivo podem receber menos estímulos. Tanto o ambiente físico quanto o socialpessoal em que a criança se desenvolve estão diretamente relacionados ao desenvolvimento neuropsicomotor, sendo que a criança que permanece em ambientes mais ricos em estímulos pode vivenciar mais experiências para desenvolver novas habilidades motoras ${ }^{23,24}$. Estudos futuros devem incluir crianças de escolas privadas, a fim de comparar o desempenho de crianças com diferentes tipos de oportunidades e estímulos, e verificar se há diferenças no desempenho motor de acordo com o nível socioeconômico.

Apesar de apontar dados importantes sobre as dificuldades motoras de crianças da região nordeste do país, o presente estudo tem limitações. A principal delas foi o uso exclusivo de questionários auto-respondidos pelos pais ou responsáveis legais. Portney e Watkins ${ }^{25}$ apontam como desvantagens do uso de questionários o fato de que os resultados obtidos podem sofrer influência do entendimento ou interpretação equivocada das questões ou opções de resposta. Outra limitação é o fato de o avaliador desconhecer a acurácia e motivação de quem responde o questionário ${ }^{25}$. Estudos futuros devem incluir, além de questionários, avaliação da criança com o uso de instrumentos padronizados, como o MABC-2.

\section{CONSIDERAÇÕES FINAIS}

Os resultados apontam prevalência considerável $(47,2 \%)$ de problemas de coordenação motora em crianças de 7 e 8 anos, estudantes de escolas da rede pública de João Pessoa/PB, além de associação das dificuldades de coordenação motora com TDAH e com nível socioeconômico. Considerando o impacto que as dificuldades motoras e o TDAH podem apresentar no desempenho da criança em casa e na escola, é essencial que essas crianças sejam diagnosticadas e recebam tratamento adequado o mais precocemente possível, para minimizar problemas secundários como depressão, baixa autoestima, baixa tolerância à frustração, etc. Além disso, a associação de dificuldades motoras com baixo nível socioeconômico aponta a necessidade de ampliar a oferta de estímulos e oportunidades de aprendizagem motora para crianças de famílias em risco social.

Agradecimentos: Agradecemos a todas as escolas, pais, professores e crianças de João Pessoa, que colaboraram para ser possível a realização deste estudo.

\section{REFERÊNCIAS}

1. American Psychiatric Association. Developmental Coordination Disorder. Diagnostic and statistical manual of mental disorder (DSM-5). 5th ed. Arlington; 2013. p.74.

2. Siqueira CM, Giannetti JG. Mau desempenho escolar: uma visão atual. Rev Assoc Med Bras. 2011;57(1):78-87. doi: http://dx.doi.org/10.1590/S0104-42302011000100021.

3. Kirby A, Sugden D, Purcell C. Diagnosing developmental coordination disorders. Arch Dis Child. 2014;99:292-6. doi: 10.1136/archdischild-2012-303569.

4. Cermak SA, Gubbay SS, Larkin D. What is developmental coordination disorder? In: Cermak SA, Larkin D. Developmental coordination disorder. Albany, NY: Delmar; 2002. p.2-22.

5. Hamilton SS. Evaluation of clumsiness in children. Am Fam Phys. 2002;66(8):1435-40. Available from: http://www.aafp. org/afp/2002/1015/p1435.html.

6. Segal R, Mandich A, Polatajko H. Stigma and its management: a framework for understanding social isolation of children with developmental coordination disorder. Am J Occup Ther. 2002;56(4):422-8.

7. Missiuna, C, Moll, S, King, S, King, G, Law, M. A trajectory of troubles: parents impressions of the impact of developmental coordination disorder. Phys Occup Ther Pediatr, 2007; 27(1): 87-101. doi: http://dx.doi.org/10.1080/ J006v27n01_06

8. Cairney J, Faught BE, Hay JA, Corna LM, Flouris AD. Developmental coordination disorder, age and play: a test of the divergence in activity-deficit with age hypothesis. Adapt Phys Activ Q. 2006;23:261-76. doi: http://dx.doi. org/10.1123/apaq.23.3.261.

9. Missiuna C, Cairney J, Pollock N, Campbell W, Russell DJ, Macdonald K, et al. Psychological distress in children with developmental coordination disorder and attentiondeficit hyperactivity disorder. Dev Disabil Res Rev. 2014;35(5):1198-207. doi: 10.1016/j.ridd.2014.01.007. 
Franca AS, et al. Problemas de coordenação motora e de atenção em crianças. Rev Ter Ocup Univ São Paulo. 2017 jan./abr.;28(1):86-92.

10. American Psychiatry Association. Manual diagnóstico e estatístico de transtornos mentais: DSM-IV-TR. 4a ed. Porto Alegre, RS: Artes Médicas; 2002.

11. Silva J, Beltrame TS. Indicativo de transtorno do desenvolvimento da coordenação de escolares com idade entre 7 e 10 anos. Rev Bras Cienc Esporte. 2013;35(1):3-14. doi: http://dx.doi.org/10.1590/S0101-32892013000100002.

12. França C. Desordem coordenativa desenvolvimental em criança de 7 e 8 anos de idade [dissertação]. Florianópolis: Universidade do Estado de Santa Catarina; 2008. Disponível em: http://portal. pmf.sc.gov.br/arquivos/arquivos/pdf/06_12_2011_9.02.44.bc7 39f001490d766574586814f745093.pdf.

13. Valentini NC, Coutinho MTC, Pansera SM, Santos VAP, Vieira JLL, Ramalho MH, et al. Prevalência de déficits motores e desordem coordenativa desenvolvimental em crianças da região Sul do Brasil. Rev Paul Pediatr. 2012;30(3):377-84. doi: http://dx.doi.org/10.1590/S010305822012000300011 .

14. Melo TFM. Influência da educação integral no desenvolvimento neuropsicomotor de crianças em idade escolar [monografia]. Ceilândia: Faculdade de Ceilândia da Universidade de Brasília; 2012. Disponível em: http://bdm. unb.br/handle/10483/4634.

15. Souza C, Ferreira L, Catuzzo MT, Corrêa UC. O teste $\mathrm{ABC}$ do movimento em crianças de ambientes diferentes. Rev Port Cienc Desp. 2007;7(1):36-47. Disponível em: http://www.fade.up.pt/rpcd/_arquivo/artigos_soltos/vol.7_ nr.1/1.04.pdf.

16. Cardoso AA, Magalhães LC, Rezende MB. Motor skills in brazilian children with developmental coordination disorder versus children with motor typical development. Occup Ther Int. 2014;21(4):176-85. doi: 10.1002/oti.1376.

17. Valentini NC, Ramalho MH, Oliveira MA. Movement assessment battery for children-2: translation, reliability and validity for brazilian children. Dev Disabil Res. 2014;35:73340. doi: 10.1016/j.ridd.2013.10.028.
18. Prado MSS, Magalhães LC, Wilson BN. Cross cultural adaptation of the Developmental Coordination Questionnaire for brazilian children. Braz J Phys Ther. 2009;13(3):236-46. doi: http://dx.doi.org/10.1590/S1413-35552009005000024.

19. Prado MS. Tradução e adaptação cultural do Developmental Coordination Disorder Questionnarie (DCDQ) [dissertação]. Belo Horizonte: Universidade Federal de Minas Gerais; 2007. Disponível em: http://hdl.handle.net/1843/MSMR-777JBN.

20. Associação Brasileira de Empresas de Pesquisa (ABEP). Critério de classificação econômica Brasil. São Paulo: ABEP; 2012. Disponível em: http://www.abep.org/new/ criterioBrasil.aspx acesso em 01 de junho de 2014.

21. Swanson J, Lerner M, March J, Gresham FM. Assessment and intervention for attention-deficit/hyperactivity disorder in the schools. Lessons from the MTA study. Pediatr Clin North Am. 1999;46(5):993-1009.

22. Mattos, P, Serra Pinheiro, MA, Rodhe, LA, Pinto, D. Apresentação de uma versão em português para o uso no Brasil do instrumento MTA-SNAP IV de Avaliação de sintomas de transtorno do déficit de atenção/hiperatividade e sintomas de transtorno desafiador e de oposição. Rev Psiquiatria. 2006;28(3):290-7. doi: http://dx.doi.org/10.1590/ S0101-81082006000300008.

23. Cardoso AA. Validade da Avaliação da Coordenação e Destreza Motora - ACOORDEM para crianças de 7 e 8 anos de idade [dissertação]. Belo Horizonte: Universidade Federal de Minas Gerais; 2011.

24. Cardoso AA, Magalhães LC. Análise da validade de critério da Avaliação da Coordenação e Destreza Motora ACOORDEM para crianças de 7 e 8 anos de idade. Braz J Phys Ther. 2012;16(1):16-22. doi: http://dx.doi.org/10.1590/ S1413-35552012000100004.

25. Portney LG, Watkins MP. Foundations of clinical research: applications to practice. New Jersey: Pearson Prentice Hall; 2009.

Recebido em: 28.09.16

Aceito em: 22.03.17 